$\begin{array}{cc}\text { Programa de Pós-Graduação em Engenharia de Produção - PPGEP } & \\ \text { Laboratório de Qualidade de Vida - LaQVida } & \\ \text { Universidade Tecnológica Federal do Paraná - UTFPR } & \text { REVISTA BRASILEIRA DE } \\ \text { Ponta Grossa - PR - Brasil } & \text { QUALIDADE DE VIDA }\end{array}$

DOI: $10.3895 / \mathrm{S} 2175-08582014000400001$

\title{
Método Isostretching como tratamento da dor lombar
}

\author{
Isostretching method to treat low back pain
}

\author{
Carlos Fernando Taborda \\ Faculdade Anglo-Americano - FAA - Foz do Iguaçu - Brasil \\ nandorccphn@hotmail.com \\ Gisele Maria Moschen \\ Faculdade Anglo-Americano - FAA - Foz do Iguaçu - Brasil \\ gisamoschen@hotmail.com \\ Magda Yaemi Mitsuro \\ Faculdade Anglo-Americano - FAA - Foz do Iguaçu - Brasil \\ magdanene@hotmail.com \\ Andersom Ricardo Fréz \\ Universidade Estadual do Centro-Oeste - UNICENTRO - Guarapuava - Paraná \\ frez@unicentro.br \\ Christiane Riedi Daniel \\ Universidade Estadual do Centro-Oeste - UNICENTRO - Guarapuava - Brasil \\ criedi@unicentro.br
}

\section{RESUMO}

OBJETIVO: Verificar os efeitos do método Isostretching na qualidade de vida, na intensidade da dor e na funcionalidade em sujeitos com dor lombar e determinar a influência desta técnica na mobilidade da coluna.

MÉTODOS: Participaram da pesquisa 18 sujeitos com idade média de 35,68 \pm 6,85 anos. Foram aplicadas de 12 a 15 posturas do método Isostretching, 3 vezes por semana, durante 8 semanas, totalizando 24 intervenções. Foram consideradas as variáveis: dor, avaliada pela Escala Visual Analógica e pelo Questionário de McGill; capacidade funcional, utilizando o Questionário de Incapacidade de Roland-Morris; qualidade de vida, utilizando o Questionário SF-36; flexibilidade, pelo banco de Wells; e, amplitude de movimento do tronco, utilizando a goniometria. Para a análise dos dados, utilizou-se o teste t pareado para os resultados com distribuição normal e o teste não paramétrico de Wilcoxon para dados não homogêneos.

RESULTADOS: Diferenças significativas foram observadas em ambas as avaliações da dor ( $p<0,001)$; da capacidade funcional $(\mathrm{p}<0,0001)$, da qualidade de vida $(\mathrm{p}<0,0001)$ e em todos os domínios do SF-36 $(\mathrm{p}<0,05)$, da flexibilidade $(\mathrm{p}<0,0001)$ e da amplitude de movimento lombar $(\mathrm{p}<0,05)$.

CONCLUSÃO: As 24 intervenções do método Isostretching foram eficazes para redução da dor, melhora da capacidade funcional e da qualidade de vida e aumento da flexibilidade da cadeia posterior e da mobilidade da coluna lombar.

PALAVRAS-CHAVE: Dor lombar. Modalidades de fisioterapia. Resultado de tratamento. 


\section{ABSTRACT}

OBJECTIVE: To investigate the effects of the method Isostretching quality of life, in pain intensity and function in subjects with low back pain and determine the influence of this technique in spinal flexibility.

METHODS: We studied 18 subjects with a mean age of $35.68 \pm 6.85$ years. Were applied from 12 to 15 postures Isostretching method, 3 times a week for 8 weeks, a total of 24 interventions. Variables were considered: pain assessed by Visual Analog Scale and the McGill Questionnaire, functional capacity, using the Roland Disability Questionnaire-Morris, quality of life using the SF36; flexibility, the Wells Bench, and lumbar range of motion using goniometry. Data analysis included the paired t-test, for the normal distribution, and Wilcoxon test, for the non-homogeneous data.

RESULTS: Significant differences were observed in both evaluation of pain $(p<0.001)$, functional capacity $(p<0.0001)$, quality of life $(p<0.0001)$ and in all domains of the SF-36 $(p<0.05)$, flexibility $(\mathrm{p}<0.0001)$ and lumbar range of motion $(\mathrm{p}<0.05)$.

CONCLUSION: The 24 Isostretching interventions were effective for pain reduction, improvement in functional capacity and quality of life and increased flexibility of the posterior chain and mobility of the lumbar spine.

KEYWORDS: Low back pain. Physical therapy modalities. Treatment outcome.

\section{Introdução}

A dor lombar tem demonstrado ser um achado importante na população mundial, sendo a segunda causa mais frequente de incapacidade, ultrapassada somente pela cefaleia (ABREU et al., 2008). Devido ao alto índice de improdutividade e absenteísmo no trabalho, a patologia é considerada um dos grandes problemas de saúde pública dos países industrializados. São muitas as consequências causadas pela dor lombar, destacando-se as incapacidades funcionais, sociais e familiares associadas às alterações psicológicas, comprometendo de forma significativa a qualidade de vida do paciente (GREVE, 2007).

A incidência dessa dor crônica vem aumentando nos últimos anos em função da alteração dos hábitos de vida, aumento do estresse e das cobranças no mundo corporativo (MATOS et al., 2008; OCARINO et al., 2009). No Brasil, cerca de 10 milhões de brasileiros perdem sua capacidade funcional devido a esta patologia (MACEDO; DEBIAGI; ANDRADE, 2010), sendo mais comum nos centros urbanos, acometendo cerca de $70 \%$ a $80 \%$ da população adulta, principalmente jovens ativos (GREVE, 2007; FRANÇA et al., 2008).

Existem múltiplos fatores causadores da dor lombar: micro-traumatismo, processos degenerativos do disco intervertebral, diminuição da força muscular e da amplitude de movimento (ADM), fatores psicológicos, inatividade física, hábitos de vida, causa degenerativa e alterações congênitas (FRANÇA et al., 2008).

A sobrecarga mecânica na coluna lombar leva a um comprometimento da força e resistência isométrica de músculos estabilizadores dessa região, resultando na fadiga muscular (GONÇALVES; BARBOSA, 2005). Porém, o principal fator causal da dor lombar é o desequilíbrio na força muscular, associada à redução da mobilidade da coluna e encurtamento da cadeia posterior (FREITAS; GREVE, 2008), decorrente de uma postura incorreta ou compensatória por um longo período. Além disso, posturas sustentadas requerem maior recrutamento e resistência da musculatura estabilizadora, desencadeando o alongamento prolongado e a compressão de vasos sanguíneos, que por consequência comprimem as terminações nervosas, provocando dor (KISNER; COLBY, 2009). 
Existem vários métodos utilizados pela fisioterapia para tratamento de pacientes com queixa de dor lombar relacionada ao desequilíbrio da força e da flexibilidade muscular. Entre esses métodos está o Isostretching, que tem seus princípios voltados para o alinhamento da coluna vertebral, o autocrescimento, o alongamento, o fortalecimento da musculatura estabilizadora, sendo os exercícios realizados na posição correta da coluna, respeitando suas curvas fisiológicas. A respiração e o posicionamento adequado do quadril e da coluna vertebral são fundamentais para a execução da técnica (MACEDO; DEBIAGI; ANDRADE, 2010). Além disso, a essência de sua ação ocorre por meio de um esforço da musculatura profunda, da flexibilidade, da mobilidade articular, do controle respiratório e da concentração mental (REDONDO, 2001). Desta forma, a ADM adequada e a flexibilidade melhoram a biomecânica corporal, contribuindo para o bem-estar e a qualidade de vida do indivíduo (KENDALL; MCCREARY; PROVANCE, 2007; REDONDO, 2006).

Neste contexto, a pesquisa teve como objetivo verificar os efeitos do método Isostretching na qualidade de vida, na intensidade da dor e na funcionalidade de sujeitos com queixa de dor lombar e determinar a influência desta técnica na mobilidade da coluna vertebral.

\section{Métodos}

Trata-se de um estudo experimental, sem grupo controle, realizado na Clínica-Escola de Fisitoerapia da Faculdade Anglo-Americano, em Foz do Iguaçu. A amostra da pesquisa foi composta por demanda espontânea de sujeitos que realizavam movimentos repetitivos e posturas inadequadas por longos períodos e que apresentavam queixa de dor lombar crônica, independente da profissão e da jornada de trabalho. Foram excluídos os sujeitos com diagnóstico clínico de patologia infecciosa ou inflamatória, doenças neurodegenerativas e/ou reumáticas, fraturas ou artrose; sujeitos em atendimento fisioterapêutico e os que completaram três faltas durante a intervenção proposta.

Foram avaliados 30 sujeitos adultos com idade entre 20 e 40 anos. Destes, 12 foram excluídos da pesquisa: 10 por atingirem 3 faltas e 2 por desistência, sendo um ocasionado pelo diagnóstico médico de fibromialgia após 12 atendimentos e outro por indisponibilidade de horário. Desta forma, a amostra do estudo foi composta por 18 sujeitos, sendo 3 homens e 15 mulheres, com

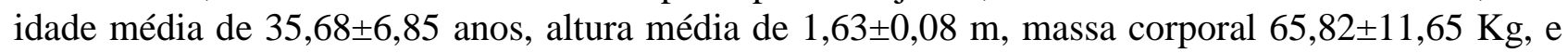

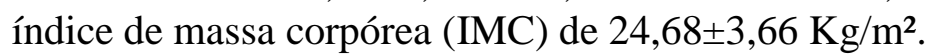

Todos receberam informações para a participação no estudo e assinaram o termo de consentimento livre e esclarecido. A pesquisa foi aprovada pelo Comitê de Ética em Pesquisa da Faculdade Assis Gurgacz (CEP/FAG), parecer 078/2011.

Foram consideradas as seguintes variáveis: dor, capacidade funcional, qualidade de vida, flexibilidade e ADM do tronco.

Para avaliar a característica e a intensidade da dor foi utilizado o Questionário de dor de McGill-BR traduzido para a língua portuguesa por Pimenta e Teixeira (1996), composto por 78 descritores, que avaliam as características da dor e sua intensidade, através de descritores subdivididos em: sensorial, afetivo, avaliativo e miscelânea. A dor também foi mensurada por meio da Escala Visual Analógica (EVA), a qual caracteriza a intensidade da dor, consistindo em um instrumento de auxílio para análise da evolução do paciente no decorrer do tratamento proposto. A EVA foi aplicada quanto ao grau de dor, contendo números de 0 a 10 , sendo que 0 significa ausência de dor e 10 é a dor máxima (TEIXEIRA; PIMENTA, 2001).

A capacidade funcional foi avaliada por meio do Questionário de Roland-Morris e Incapacidade (SARDÁ JÚNIOR et al., 2010), o qual consiste de 24 itens sobre a interferência das dores nas costas nas atividades da vida diária e da vida prática do paciente, que são assinalados se presentes no cotidiano deste.

A qualidade de vida foi avaliada utilizando o questionário Short-Form Survey, conhecido como SF-36. É um instrumento composto por 36 questões, o qual consiste em oito domínios, com escores que variam de 0 a 100, que consideram a percepção do paciente sobre a forma como sua 
saúde, bem-estar e a maneira como desempenha suas atividades de vida diária, interferem em sua qualidade de vida (CICONELLI et al., 1999).

Para avaliar a flexibilidade da cadeia posterior foi utilizado o banco de Wells. Os sujeitos sentaram de frente para o banco, apoiando os pés no banco e com extensão total dos joelhos. Com as mãos sobrepostas, flexionaram o tronco, empurrando o marcador para o mais distante possível na régua do banco. Foram realizadas três repetições para o aquecimento e a correção, e uma única execução para obter o resultado (DANTAS, 2005).

Para avaliar a ADM do tronco foi utilizada a goniometria e foram considerados os movimentos de flexão, extensão, flexão lateral e rotação do tranco (MARQUES, 2003).

Após a avaliação, os sujeitos iniciaram o tratamento que consistiu de 24 atendimentos com o método Isostretching, aplicado 3 vezes por semana, durante 8 semanas, com duração aproximada de 45 minutos. Para otimização do espaço físico, os atendimentos foram realizados em dois horários distintos, tendo nove sujeitos em cada horário, não caracterizando subgrupos, já que todos os pacientes foram atendidos pelos mesmos pesquisadores e, consequentemente, as mesmas posturas foram aplicadas nos dois horários.

Nos primeiros atendimentos os voluntários receberam informações básicas sobre a prática do método, como: a posição da coluna, movimentos da pelve (anteroversão e retroversão), o controle respiratório profundo e forçado durante a expiração, a contração isométrica dos estabilizadores da coluna, da musculatura dos membros inferiores e o autocrescimento. As posturas foram realizadas em decúbito dorsal, sentadas e ortostáticas, conforme a descrição do criador do método (REDONDO, 2006), com o pesquisador dando estímulos verbais e táteis para a realização correta dos exercícios. Cada postura foi mantida num tempo de três expirações forçadas a partir da posição correta, sendo repetida três vezes com intervalo de um minuto entre a mudança de postura.

Nas 6 primeiras semanas foram realizadas 12 posturas de Isostretching sendo sempre iniciado por 4 posturas em decúbito dorsal, em seguida passando para 4 posturas sentadas e finalizando com as últimas 4 em ortostáse.

Como houve melhora no desempenho na realização dos exercícios e na redução da dor, avaliada em todas as intervenções utilizando a EVA, nas 2 últimas semanas foram aplicadas 15 posturas em cada atendimento (5 em cada posição), aumentando o tempo da contração e as repetições das expirações prolongadas como forma de progressão do tratamento.

As reavaliações ocorreram no $24^{\circ}$ atendimento, nos mesmos critérios da avaliação.

Foi realizada análise estatística descritiva para calcular a média e o desvio padrão dos resultados. Utilizou-se o teste de normalidade Kolmogorov-Smirnov para análise da distribuição da amostra. Após esta análise utilizou-se o teste t pareado para os resultados com distribuição normal e o teste não paramétrico de Wilcoxon para dados não homogêneos.

$\mathrm{O}$ nível de significância foi estabelecido em $\mathrm{p}<0,05$. Os testes foram realizados com o uso do software Graphpad Instat versão 3.0.

\section{Resultados}

Por meio do questionário de Roland-Morris, foi observada um melhora funcional, apresentando redução do escore de $3,94 \pm 3,46$ para $1,22 \pm 2,13$ pontos $(\mathrm{p}<0,0001)$. Em relação à dor, também foram observadas reduções na intensidade e na característica após a intervenção (Tabela 1).

Tabela 1 - Intensidade e característica da dor pré e pós-intervenção

\begin{tabular}{cccc}
\hline \multicolumn{1}{c}{ Variáveis } & Pré & Pós & p \\
\hline EVA & $3,09 \pm 2,32$ & $0,06 \pm 0,24$ & $<0,0001^{*}$ \\
Questionário de McGill & & & \\
Sensorial & $17,61 \pm 7,61$ & $4,67 \pm 7,44$ & $<0,0001^{*}$ \\
Afetivo & $4,06 \pm 2,90$ & $1,06 \pm 1,92$ & $0,0001^{*}$ \\
Avaliativo & $1,67 \pm 1,24$ & $0,39 \pm 0,78$ & $0,0005^{*}$ \\
Miscelânea & $4,78 \pm 3,52$ & $1,22 \pm 2,21$ & $0,0002^{*}$ \\
\hline \multicolumn{4}{r}{ Foxtremamente significativo } \\
& Fonte: Autoria própria (2012).
\end{tabular}


$\mathrm{Na}$ avaliação da qualidade de vida, com o questionário SF-36, verificou-se um incremento no índice geral da qualidade de vida, tendo como escore inicial 58,23 29,62 e, após a intervenção, $82,16 \pm 21,09$ ( $\mathrm{p}<0,0001)$. Todos os componentes e domínios do SF-36 também apresentaram melhora significativa (Tabela 2).

Tabela 2 - Qualidade de vida pré e pós-intervenção (escores do SF-36)

\begin{tabular}{|c|c|c|c|}
\hline Domínios & Pré & Pós & $\mathbf{P}$ \\
\hline Componente físico & $58,86 \pm 27,89$ & $79,14 \pm 23,20$ & $<0,0001^{*}$ \\
\hline Capacidade Funcional & $78,06 \pm 14,77$ & $86,39 \pm 22,54$ & $0,0110^{* *}$ \\
\hline Aspectos Físicos & $45,83 \pm 40,45$ & $87,50 \pm 28,76$ & $0,0134^{* *}$ \\
\hline Dor & $51,89 \pm 24,21$ & $73,94 \pm 21,14$ & $0,0002^{*}$ \\
\hline Estado Geral de Saúde & $59,67 \pm 13,65$ & $68,72 \pm 13,89$ & $0,0034^{* *}$ \\
\hline Componente mental & $57,61 \pm 31,44$ & $85,18 \pm 18,41$ & $<0,0001^{*}$ \\
\hline Vitalidade & $53,33 \pm 21,00$ & $83,06 \pm 14,36$ & $<0,0001^{*}$ \\
\hline Aspectos Sociais & $70,14 \pm 27,83$ & $87,50 \pm 22,28$ & $0,0093^{* *}$ \\
\hline Aspectos Emocionais & $46,29 \pm 45,93$ & $81,48 \pm 23,49$ & $0,0020^{* *}$ \\
\hline Saúde Mental & $60,67 \pm 21,75$ & $88,67 \pm 11,08$ & $<0,0001^{*}$ \\
\hline
\end{tabular}

A flexibilidade da cadeia posterior apresentou aumento extremamente significativo ( $\mathrm{p}<0,0001$ ), passando de $275,83 \pm 76,32 \mathrm{~cm}$ para $393,22 \pm 68,38$ após a aplicação do método Isostretching. Aumento também observado na ADM da coluna lombar (Tabela 3).

Tabela 3 - ADM da coluna lombar pré e pós-intervenção (em graus)

\begin{tabular}{lccc}
\hline \multicolumn{1}{c}{ Movimentos } & Pré & Pós & p \\
\hline Extensão & $23,06 \pm 5,60$ & $30,00 \pm 7,47$ & $0,0004^{*}$ \\
Flexão & $90,11 \pm 13,02$ & $102,89 \pm 12,36$ & $<0,0001^{*}$ \\
Rotação direita & $25,22 \pm 9,35$ & $32,72 \pm 11,17$ & $0,0041^{* *}$ \\
Rotação esquerda & $27,56 \pm 13,28$ & $34,33 \pm 14,62$ & $0,0046^{* *}$ \\
Flexão lateral direita & $25,39 \pm 10,04$ & $30,28 \pm 10,87$ & $0,0316^{* *}$ \\
Flexão lateral esquerda & $24,56 \pm 5,45$ & $31,78 \pm 10,28$ & $0,0008^{*}$ \\
\hline
\end{tabular}

extremamente significativo; ${ }^{* *}$ significativo

Fonte: Autoria própria (2012).

\section{Discussão}

Como a prevalência dos distúrbios osteomusculares continua crescendo entre a população de trabalhadores e o problema torna-se cada vez mais preocupante dentro de um cenário mundial de promoção de saúde laboral (BRANDÃO; HORTA; TOMASI, 2005), no presente estudo optou-se pela seleção da amostra por sujeitos que realizavam movimentos repetitivos e posturas inadequadas por longos períodos e que apresentavam queixa de dor lombar crônica, independente da profissão e/ou da jornada de trabalho.

Em relação ao tratamento da dor lombar, existem evidências de que atividades relacionadas ao controle motor, implementadas como programas de tratamento incluindo fisioterapia e exercícios (KO, 2007), terapia manual e reeducação (MOSELEY, 2002), ou contendo apenas terapia manual (NIEMISTÖ et al., 2003), sejam mais efetivas que o tratamento medicamentoso.

Fatos semelhantes foram observados em revisões sistemáticas (FURLAN et al., 2009; FERREIRA et al. 2009), nas quais os autores observaram que os exercícios terapêuticos são mais efetivos que a usual abordagem médica no tratamento da dor lombar crônica, com o objetivo de diminuir a dor, melhorar a função e a qualidade de vida, inclusive como prevenção de episódios de recorrência. Sabe-se, também, que a prática de exercícios tem sido fundamental no tratamento e na prevenção de dores lombares, além da importância no aumento da flexibilidade e da força muscular (MACEDO; MOTTER; KIRSCHEN, 2011). 
Como recurso terapêutico, a escolha do método Isostretching aconteceu em função da efetividade no tratamento da dor lombar crônica e na incapacidade funcional, na melhora da força muscular e da flexibilidade e na correção vícios posturais (MORAES; MATEUS, 2005). O método Isostretching enfatiza a endurance dos músculos estáticos por meio de contrações isométricas e, ao mesmo tempo, exige o controle respiratório e o alongamento de grandes grupos musculares, aumentando assim, a flexibilidade e a capacidade funcional em sujeitos com queixa de dor lombar (LOPES et al., 2006). Além destes fatores, o método também melhora a execução das atividades laborais e promove uma consciência corporal nas atividades de vida diária (REDONDO, 2001; REDONDO, 2006).

No presente estudo todos os sujeitos apresentaram ganhos físicos e funcionais após a aplicação do método Isostretching, o qual se demonstrou efetivo em todas as variáveis analisadas: dor, capacidade funcional, qualidade de vida, flexibilidade e ADM do tronco.

Bons resultados do método para redução da dor lombar também foram observados em outros estudos (MACEDO; DEBIAGI; ANDRADE, 2010; LOPES et al., 2006), tanto na comparação pré e pós-intervenção, quanto com o grupo controle (MACEDO; DEBIAGI; ANDRADE, 2010). Porém, com número de atendimentos distintos: 20 (MACEDO; DEBIAGI; ANDRADE, 2010), 12 (LOPES et al., 2006) e na presente pesquisa 24, mas em todos os estudos a frequência semanal foi de 3 atendimentos.

Estes mesmos estudos (MACEDO; DEBIAGI; ANDRADE, 2010; LOPES et al., 2006) também avaliaram a capacidade funcional por meio do Questionário de Roland-Morris e Incapacidade e, como na presente pesquisa, demonstraram reduções significativas nos escores após a intervenção. Já em uma pesquisa com idosos (CARVALHO; ASSINI, 2008), os autores utilizaram o teste de caminhada de seis minutos para avaliar a capacidade funcional após a intervenção com o método Isostretching, e concluíram ser um método eficiente para amenizar as incapacidades decorrentes do envelhecimento.

Em relação à qualidade de vida, os resultados observados foram semelhantes a outros estudos que utilizaram o método Isostretching para tratar lombalgia. No estudo de Adorno e BrasilNeto (2013), 24 intervenções do método Isostretching durante 3 meses melhorou a qualidade de vida em todos os domínios do SF-36, sendo este método mais eficaz que a Reeducação Postural Global (RPG) no seguimento de 2 meses. Já no estudo de Macedo e Briganó (2009), utilizando o método Isostretching por dez semanas, foi possível melhorar a dor e da qualidade de vida em todos os domínios do SF-36, porém, o método foi associado à terapia manual e a cinesioterapia. Acreditase que a redução da dor e a melhora da qualidade de vida estejam relacionadas ao aumento da resistência muscular de abdominais, glúteo máximo e extensores de tronco, uma vez que os trabalhos de força e resistência muscular são importantes para indivíduos com dor lombar (MACEDO; DEBIAGI; ANDRADE, 2010). Considerando outra queixa de trabalhadores, a síndrome do impacto, Barbosa et al. (2012) demonstraram que duas intervenções por semana, durante seis semanas com o método Isostretching melhorou a qualidade de vida em quase todos os domínios do questionário SF-36: capacidade funcional, aspectos funcionais, dor, vitalidade, aspectos sociais, aspectos emocionais e saúde mental.

A variável mais avaliada entre os estudos envolvendo o método Isostretching é a flexibilidade da cadeia posterior. $\mathrm{O}$ uso em excesso de grupos musculares sem visar sua completa amplitude gera um encurtamento da musculatura sobrecarregando as articulações limitando assim sua flexibilidade e gerando dores (MACEDO; DEBIAGI; ANDRADE, 2010). Uma boa flexibilidade ajuda a evitar lesões, reduzir as dores musculares e aumentar o nível de eficiência em todas as atividades físicas, além de melhorar a qualidade de vida e a independência funcional (ARNAUD; CORREA, 2000). Como no presente estudo, o método Isostretching demonstrou-se efetivo para melhora da flexibilidade (LOPES et al., 2006; ARNAUD; CORREA, 2000; MONTERASO et al., 2009), independente da forma e da técnica de mensuração.

Já em relação à ADM, os resultados também foram semelhantes a outro estudo (MACEDO; MOTTER; KIRSCHEN, 2011), o qual considerou o método Isostretching indicado para pacientes com diminuição da mobilidade da região lombar. Estes mesmos autores (MACEDO; MOTTER; 
KIRSCHEN, 2011) observaram que 24 atendimentos, 2 vezes por semana, usando uma média de 12 posturas por atendimento, foram eficazes para melhorar todos os movimentos da coluna lombar.

Além das variáveis analisadas na presente pesquisa, na literatura foi possível observar a efetividade do método Isostretching para aumentar a resistência dos músculos abdominais, glúteo máximo e extensores do tronco de pacientes com dor lombar (MACEDO; DEBIAGI; ANDRADE, 2010). Bons resultados também foram demonstrados para o alinhamento da coluna vertebral (LONGATO et al., 2011; DURANTE; VASCONCELOS, 2009), porém, com melhora eficiente apenas para as alterações no plano sagital, não sendo tão eficaz no tratamento das assimetrias no plano frontal (REDONDO, 2006; MONTE-RASO et al., 2009). Outros fatores observados após a prática do método Isostretching foram a melhora na conscientização da imagem corporal, melhorando, assim, a autoestima (ARNAUD; CORREA, 2000), melhora do padrão da marcha em idosos (SANGLARD; PEREIRA, 2005) e a redução das alterações de equilíbrio em sujeitos com amputação transfemoral (LONGATO et al., 2011).

Além do método Isostretching, outras técnicas também demonstraram ser eficientes na redução da dor e da incapacidade funcional, como a estabilização segmentar, que também utiliza exercícios com o máximo de recrutamento dos músculos transverso do abdômen, multífidos e diafragma (FRANÇA et al., 2008; BORDIAK; SILVA, 2011; DURANTE; VASCONCELOS, 2009; SANTOS et al., 2012). A RPG também tem influência na dor e no aumento da flexibilidade, e na melhora da força muscular respiratória, expansibilidade torácica e mobilidade toracoabdominal (TEODORI et al., 2011). Os exercícios de fortalecimento dos extensores do tronco em pessoas com dor lombar crônica favorecem ao aumento da flexibilidade e mobilidade da coluna lombar (DIAS; BRECH; NIGRO FILHO, 2011).

O método Isostretching é de difícil execução, pois exige contrações musculares constantes e tensões musculares, limitando os movimentos compensatórios e exigindo o máximo de concentração e controle respiratório por parte dos participantes (REDONDO, 2006), na amostra da presente pesquisa a dificuldade para realização das posturas foi observada apenas nos primeiros dias.

Em relação aos procedimentos de avaliação, optou-se pelo uso de instrumentos validados para a população brasileira e procedimentos rotineiros na prática avaliativa da fisioterapia. Como a maioria dos sujeitos com queixa de dor lombar também apresentam fraqueza dos músculos profundos (GOUVEIA; GOUVEIA, 2008), podem ser utilizados testes eletromiográficos para melhor detalhar a contração isométrica desta musculatura (CARVALHO; RIBEIRO; RIBEIRO, 2011); ou ainda, o uso da unidade de biofeedback pressórico (Stabilizer) (COSTA et al., 2004). Porém, para execução da presente pesquisa tais ferramentas não estavam disponibilizadas para avaliação da musculatura profunda.

Seguindo a linha das pesquisas citadas acima, durante a realização deste estudo procurouse manter todos os procedimentos de maneira padronizada para evitar possíveis erros de execução dos testes, incluindo o posicionamento dos voluntários. Além disso, numa tentativa de reduzir possíveis vieses da aplicação do método, todos os pesquisadores tiveram formação em Isostretching com o criador da técnica Bernard Redondo. Entretanto, o estudo apresenta como limitação a não avaliação da conscientização corporal e o comportamento postural pré e pós-intervenção, fato que após o término da pesquisa foi considerado importante, pois o método também pode ser autoaplicado no ambiente de trabalho. Além disso, não foi dado seguimento para analisar os efeitos a médio e longo prazo.

\section{Conclusão}

Para uma amostra de pessoas, com idade entre 20 e 40 anos, que realizavam movimentos repetitivos e posturas inadequadas por longos períodos e apresentavam queixa de dor lombar crônica, 24 atendimentos utilizando o método Isostretching, aplicado três vezes por semana mostrou-se efetivo na redução da dor lombar, melhora da capacidade funcional, da qualidade de vida, da flexibilidade e da ADM do tronco. 


\section{Referências}

ABREU, A. M.; FARIA, C. D. C. M.; CARDOSO, S. M. V.; SALMELA, L. F. T. Versão brasileira do fear avoidance beliefs questionnaire. Cadernos de Saúde Pública, v. 24, n. 3, p. 615-623, 2008. crossef

ADORNO, M. L. G. R.; BRASIL-NETO, J. P. Avaliação da qualidade de vida com o instrumento SF-36 em lombalgia crônica. Acta Ortopédica Brasileira, v. 21, n. 4, p. 202-207, 2013. rossef

ARNAUD, A. S.; CORREA, A. F. O reequilíbrio postural através do método de isostretching. 2000. 190 f. Monografia (Graduação em Fisioterapia) - Setor de Ciências Biológicas e da Saúde, Universidade do Estado do Pará, Belém, 2000.

BARBOSA, É. C., PERES, C. M., LUCCA, S. R., OLIVEIRA, J. I. Melhora na qualide de vida e da dor referida em trabalhadores com síndorme do impacto após aplicação do método Isostretching. Acta Fisiátrica, v. 19, n. 3, p. 178-183, 2012. Crossef

BORDIAK, F. C.; SILVA, E. B. O core training como programa de exercícios de estabilização. Fisioterapia Brasil, v. 12, n. 3, p. 219-223, 2011.

BRANDÃO, A. G.; HORTA, B. L.; TOMASI, E. Sintomas de distúrbios osteomusculares em bancários de Pelotas e região: prevalência e fatores associados. Revista Brasileira de Epidemiologia, v. 8, n. 3, p. 295-305, 2005. Crossef

CARVAlHO, A. R.; ASSINI, T. C. K. A. Aprimoramento da capacidade funcional de idosos submetidos a uma intervenção por isostretching. Revista Brasileira de Fisioterapia, v. 12, n. 4, p. 268-273, 2008. Crossef

CARVALHO, J.; RIBEIRO, G.; RIBEIRO, M. M. Atividade eletromiográfica da musculatura associada à expiração forçada. Revista Brasielira de Fisiologia do Exercício, v. 10, n. 1, p. 23-30, 2011.

CICONELLI, R. M.; FERRAZ, M. B.; SANTOS, W.; MEINÃO, I.; QUARESMA, R. M. Tradução para o português e validação do questionário genérico de avaliação de qualidade de vida SF-36. Revista Brasileira de Reumatologia, v. 39, n. 3, p. 580-588, 1999.

COSTA, L. O. P.; COSTA, L. C. M.; CANÇADO, R. L.; OLIVEIRA, W. M.; FERREIRA, P. H. Confiabilidade do teste palpatório e da unidade de biofeedback pressórico na ativação do músculo transverso abdominal em indivíduos normais. Acta Fisiátrica, v. 11, n. 3, p. 101-105, 2004.

DANTAS, E. H. M. Alongamento e flexionamento. 5. ed. Rio de Janeiro: Shape, 2005.

DIAS, L. B.; BRECH, G. C.; NIGRO FILHO, A. Exercícios de fortalecimento dos extensores do tronco no tratamento da lombalgia crônica. Fisioterapia Brasil, v. 12, n. 3, p. 193-199, 2011.

DURANTE, H.; VASCONCELOS, E. C. L. M. Comparação do método isostretching e cinesioterapia convencional do tratamento da lombalgia. Semina, v. 30, n. 1, p. 83-90, 2009.

FERREIRA, M. C.; PENIDO, H.; AUN, A.; FERREIRA, P.; FERREIRA, M. L.; OLIVEIRA, V. C. Eficácia dos exercícios de controle motor na dor lombopélvica: uma revisão sistemática. Fisioterapia e Pesquisa, v. 16, n. 4, p. 374-379, 2009. Crossef 
FRANÇA, F. J. R.; BURKE, T. N.; CLARET, D. C.; MARQUES, A. P. Estabilização segmentar da coluna lombar nas lombalgias: uma revisão bibliográfica e um programa de exercícios. Fisioterapia e Pesquisa, v. 15, n. 2, p. 200-206, 2008. Crossef

FREITAS, C. D.; GREVE, J. M. D. A. Estudo comparativo entre exercícios com dinamômetro isocinético e bola terapêutica na lombalgia crônica de origem mecânica. Fisioterapia e Pesquisa, v. 15 , n. 4 , p. 380-386, 2008. Crossef

FURLAN, A. D.; PENNICK, V.; BOMBARDIER, C.; VAN TULDER, M. 2009 update method guidelines for systematic reviews in the Cochrane Collaboration Back Review Group. Spine, v. 34, n. 18, p. 1929-1941, 2009. Crossef

GONÇALVES, M.; BARBOSA, F. S. S. Análise de parâmetros de força e resistência dos músculos eretores da espinha lombar durante a realização de exercício isométrico em diferentes níveis de esforço. Revista Brasielira de Medicina do Esporte, v. 11, n. 2, p. 109-114, 2005. Crossef

GOUVEIA, K. M. C.; GOUVEIA, E. C. O músculo transverso abdominal e sua função de estabilização da coluna lombar. Fisioterapia em Movimento, v. 21, n. 3, p. 45-50, 2008.

GREVE, J. M. D. A. Tratado de medicina de reabilitação. São Paulo: Roca, 2007.

KENDALl, F. P.; MCCREARY, E. K.; PROVANCE, P. G. Músculos provas e funções. 5. ed. São Paulo: Manole, 2007.

KISNER, C.; COLBY, L. A. Exercícios terapêuticos: fundamentos e técnicas. 5. ed. Barueri: Manole, 2009.

KO, J. K. Comparing the effects of drug therapy, physical therapy, and exercise on pain, disability, and depression in patients with chronic low back pain. Taehan Kanho Hakhoe Chi, v. 37, n. 5, p. 645-654, 2007.

LONGATO, M. W.; CASTRO, P. R.; KELLER, K. C.; RIBAS, D. I. R. Efeito do isostretching no equilíbrio de indivíduos amputados: um estudo de caso. Fisioterapia em Movimento, v. 24, n. 4, p. 689-696, 2011.

LOPES, P. M.; MACKERT, T. C.; YAU, M. C. H.; FACCI, L. M. Isostretching no tratamento da lombalgia crônica. Fisioterapia Brasil, v. 7, n. 2, p. 99-103, 2006.

MACEDO, A. C. B.; MOTTER, A. A.; KIRSCHEN, S. Avaliação da mobilidade da coluna lombar após aplicação do isostretching. Fisioterapia Brasil, v. 12, n. 1, p. 31-36, 2011.

MACEDO, C. S. G.; DEBIAGI, P. C.; ANDRADE, F. M. Efeito do isostretching na resistência muscular de abdominais, glúteo máximo e extensores de tronco, incapacidade e dor em pacientes com lombalgia. Fisioterapia em Movimento, v. 23, n. 1, p. 113-120, 2010. rossef

MACEDO C. S. G.; BRIGANÓ J. U. Terapia manual e cinesioterapia na dor, incapacidade e qualidade de vida de indivíduos com lombalgia. Revista Espaço para a Saúde, v. 10, n. 2, p. 1-6, 2009.

MARQUES, A. P. Manual de goniometria. 2. ed. São Paulo: Manole, 2003. 
MATOS, M. G.; HenNington, E. A.; HOEFEL, A. L.; COSTA, J. S. D. Dor lombar em usuários de um plano de saúde: prevalência e fatores associados. Cadernos de Saúde Pública, v. 24, n. 9 , p. 2115-2122, 2008. Crossef

MONTE-RASO, V. V.; FERREIRA, P. A.; CARVALHO, M. S.; RODRIGUES, J. G.; MARTINS, C. C.; LUNES, D. H. Efeito da técnica isostretching no equilíbrio postural. Fisioterapia e Pesquisa, v. 16, n. 2, p. 137-142, 2009. Crossef

MORAES, S. M. S.; MATEUS, E. C. L. O método isostretching no tratamento da hipercifose torácica. Fisioterapia Brasil, v. 6, n. 4, p. 311-313, 2005.

MOSELEY, L. Combined physiotherapy and education is efficacious for chronic low-back pain. Australian Journal of Physiotherapy, v. 48, n. 4, p. 297-301, 2002. rossef

NIEMISTÖ, L.; LAHTINEN-SUOPANKI, T.; RISSANEN, P.; LINDGREN, K. A.; SARNA, S.; HURRI, H. A randomized trial of combined manipulation, stabilizing exercises, and physician consultation compared to physician consultation alone for chronic low-back pain. Spine, v. 28, n. 19, p. 2185-2191, 2003. Crossef

OCARINO, J. M.; GONLÇALVES, G. G. P.; VAZ, D. V.; CABRAL, A. A. V.; PORTO, J. V.; SILVA, M. T. Correlação entre um questionário de desempenho funcional e testes de capacidade física em pacientes com lombalgias Revista Brasielira de Fisioterapia, v. 13, n. 4, p. 343-349, 2009.

PIMENTA, C. A.; TEIXEIRA, M. J. Questionário de dor McGill: proposta de adaptação para a língua portuguesa. Revista da Escola de Enfermagem da USP, v. 30, n. 3, p. 473-483, 1996.

REDONDO, B. Isostretching: a ginástica da coluna. Piracicaba: Skin Direct Store, 2001.

REDONDO, B. Isostretching: a reeducação da coluna. 2. ed. Piracicaba: CSBM Produtos Médicos, 2006.

SANGLARD, R. C, F.; PEREIRA, J. S. A influência do isostretching nas alterações dos parâmetros da marcha em idosos. Fisioterapia Brasil, v. 6, n. 4, p. 255-260, 2005.

SANTOS, A. M.; RUARO, M. B.; FRÉZ, A. R.; RUARO, J. A. Stabilizing muscles of the lumbar spine strengthening in the treatment of low back pain. Fiep Boulletin, v. 82, special edition, p. 144 $148,2012$.

SARDÁ JÚNIOR, J. J.; NICHOLAS, M. K.; PIMENTA, C. A. M.; ASGHARI, A.; THIEME, A. L. Validação do questionário de incapacidade Roland Morris para dor em geral. Revista Dor, v. 11, n. 1, p. 28-36, 2010.

TEIXEIRA, M. J.; PIMENTA, C. A. M. Avaliação do doente com dor. In: TEIXEIRA, M. J.; FIGUEIRÓ, J. A. B. Dor: epidemiologia , fisiopatologia, avaliação, síndromes dolorosas e tratamento. São Paulo: Moreira Júnior, 2001.

TEODORI, R. M.; NEGRI, J. R.; CRUZ, M. C.; MARQUES, A. P. Reeducação postural global: uma revisão da literatura. Revista Brasileira de Fisioterapia, v. 15, n. 3, p. 185-189, 2011. Crossef 